\title{
Towards Probabilistic Bitemporal Knowledge Graphs
}

\author{
Melisachew Wudage Chekol \\ Data and Web Science Group \\ University of Mannheim \\ mel@informatik.uni-mannheim.de
}

\author{
Heiner Stuckenschmidt \\ Data and Web Science Group \\ University of Mannheim \\ heiner@informatik.uni-mannheim.de
}

\begin{abstract}
The emergence of open information extraction as a tool for constructing and expanding knowledge graphs has aided the growth of temporal data, for instance, YAGO, NELL and Wikidata. While YAGO and Wikidata maintain the valid time of facts, NELL records the time point at which a fact is retrieved from some Web corpora. Collectively, these knowledge graphs (KGs) store facts extracted from Wikipedia and other sources. Due to the imprecise nature of the extraction tools that are used to build and expand KGs, such as NELL, the facts in the KGs are weighted (a confidence value representing the correctness of a fact). Additionally, NELL can be considered as a transaction time KG because every fact is associated with extraction date. On the other hand, YAGO and Wikidata use the valid time model because they only maintain facts together with their validity time (temporal scope). In this paper, we propose a bitemporal model (that combines transaction and valid time models) for maintaining and querying probabilistic temporal knowledge graphs. We report our evaluation results of the proposed approach.
\end{abstract}

ACM Reference Format:

Melisachew Wudage Chekol and Heiner Stuckenschmidt. 2018. Towards Probabilistic Bitemporal Knowledge Graphs. In WWW'18 Companion: The 2018 Web Conference Companion, April 23-27, 2018, Lyon, France. ACM, New York, NY, USA, 6 pages. https://doi.org/10.1145/3184558.3191637

\section{INTRODUCTION}

Temporal databases have been studied extensively (see survey [22]). Recently, support for temporal data from database vendors, such as Teradata, Oracle DB, IBM DB2, PostgreSQL, and so on, has been growing. On the other hand, probabilistic temporal databases have been given little attention $[7,10,18]$ and even less for probabilistic temporal knowledge graphs [5, 11]. Several Web knowledge graphs, such as YAGO [14], Wikidata [24], NELL [3], and DBpedia [1], already contain temporal data. In particular, NELL contains probabilistic temporal data where each fact in the knowledge base is associated with a transaction time (the time when a fact is extracted and stored in the knowledge base). Besides, the emergence of open information extraction as a tool for constructing and expanding knowledge graphs (KGs) has aided the growth of temporal data $[20,23]$. With respect to knowledge graphs, while maintaining the valid time of facts is clearly relevant, having transaction time is somewhat arguable. In addition to valid time support, maintaining the transaction time of facts is important because never-ending

This paper is published under the Creative Commons Attribution 4.0 International (CC BY 4.0) license. Authors reserve their rights to disseminate the work on their personal and corporate Web sites with the appropriate attribution.

WWW'18 Companion, April 23-27, 2018, Lyon, France

() 2018 IW3C2 (International World Wide Web Conference Committee), published under Creative Commons CC BY 4.0 License.

ACM ISBN 978-1-4503-5640-4/18/04.

https://doi.org/10.1145/3184558.3191637 open information extraction systems such NELL, Google's Knowledge Vault [9], Microsoft's Satori continuously learn new facts from the Web and it is important to record the date on which a fact is learned. This is evident from the NELL knowledge graph as they keep track of learned dates in terms of iterations. Hence, transaction times represent the date on which a fact is extracted or recorded. The valid time indicates the time period on which a fact is considered valid or true. Furthermore, when such facts are learned/extracted from open text, they are associated with some confidence score. We need a model that supports these three desirable aspects (transaction time, validity time, and confidence scores). Thus, in this work, we propose a framework for representing and querying probabilistic bitemporal knowledge graphs.

Probabilistic graphical models have been widely used to reason about facts extracted at Web scale using a combination of handcrafted and extracted inference rules [21]. In particular, Markov logic networks (MLNs) can be used to deal with temporal relations in open information extraction [17] or check the consistency of knowledge bases [5, 6]. MLNs extend first order logic with uncertainty and are considered as templates for defining Markov networks. Another important probabilistic programming language is ProbLog [15] which is a probabilistic extension of Prolog. Like MLNs, it has been used for reasoning in probabilistic knowledge bases. In both MLNs and ProbLog, there are two important reasoning tasks marginal and most probable explanation (MPE) inference. The former computes the probability of a set of random variables (temporal facts in our setting) whereas the later computes the most probable, consistent and conflict-free world (temporal knowledge graph). In this work, we propose a probabilistic database model for bitemporal knowledge graphs.

A relevant problem in probabilistic temporal KGs is temporal coalescing. Temporal coalescing is the process of merging facts with identical non-temporal arguments and adjacent or overlapping time-intervals. This problem has been throughly studied in the database community in a non-probabilistic setting (look for instance [2]). In this paper we investigate two approaches for coalescing probabilistic temporal facts. Overall, the contributions of this paper are: (i) we propose a probabilistic bitemporal model for Web knowledge graphs, (ii) we study temporal coalescing in a probabilistic setting, (iii) we provide query rewriting for marginal and MPE inference tasks, and (iv) we provide experimental results of the proposed approach over the Wikidata KG.

Outline. The outline of this paper is as follows: next we provide a motivating example. In Section 2 we discuss the state of the art, followed by the introduction of bitemporal knowledge graphs in Section 3. We present our main contribution in Section 4. Preliminary experimental results are presented in Section 5; and finally we present the discussion of related work (Section 6) along with concluding remarks and outlook (Section 7). 


\begin{tabular}{|lll|}
\hline NELL: learned fact & Date learned & Confidence \\
\hline (fernando-torres, type, athlete) & $12 / 01 / 2010$ & 1.0 \\
(fernando-torres, home-stadium, anfield) & $10 / 11 / 2015$ & 1.0 \\
(fernando-torres, playsfor, chelsea) & $09 / 02 / 2017$ & 0.969 \\
(fernando-torres, playsfor, spain) & $08 / 08 / 2011$ & 0.875 \\
\hline Wikidata: extracted fact & Valid time & Confidence \\
\hline (fernando-torres, playsfor, atleticoMadrid) & {$[2001,2007)$} & 1.0 \\
(fernando-torres, playsfor, liverpool) & {$[2007,2011)$} & 1.0 \\
(fernando-torres, playsfor, chelsea) & {$[2011,2015)$} & 1.0 \\
(fernando-torres, playsfor, atleticoMadrid) & {$[2016$, now) } & 1.0 \\
\hline
\end{tabular}

Table 1: NELL and Wikidata representations of the career of Fernando Torres.

\subsection{Motivation}

To motivate the purpose of this study we rely on two prominent knowledge bases: NELL and Wikidata. NELL records the extraction dates of facts as shown in Table 1. For instance, it is extracted or recorded on 09/02/2017 that Fernando Torres plays for the Chelsea football club with a confidence of $96.9 \%$. This shows that NELL's representation model resembles that of transaction time from relational databases. On the other hand, Wikidata scopes temporally some of the facts, for instance, with $100 \%$ confidence Fernando Torres played for Atletico Madrid from 2001 to 2007 before rejoining them on 2016 and he is still playing for them. This shows that Wikidata uses the valid time model for modeling temporal information. However, Wikidata does not record the extraction date of facts, conversely, NELL does not maintain the valid time of facts. Thus, what is missing is a model which combines both transaction time and valid time (i.e., bitemporal). For instance, with a 0.969 probability Fernando Torres played for Chelsea football club from 2011 to 2015 . This fact is extracted (or recorded) on $09 / 02 / 2017$ as shown below:

(fernando-torres, playsfor, chelsea, [2011,2015), [09/02/2017,UC), 0.969) UC is short for until changed. When the end date of the transaction or recording date is unknown, it is denoted by UC.

In addition to probabilistic temporal facts, a knowledge base (KB) can contain (temporal) inference rules, for instance, some of the deduction rules learned from the NELL KB using Prob2FOIL are shown below: (i) if an athlete led a sports team, then she probably plays for that team; (ii) during the same time period if an athlete led a sports team and her team plays against another team, then she probably plays for that team; (iii) if an athlete plays some sport and a team plays that sport during an overlapping time period, then that athlete probably plays for that team; (iv) during some time period if an athlete plays in a league and a team plays in that league over the same time period, then she probably plays for that team.

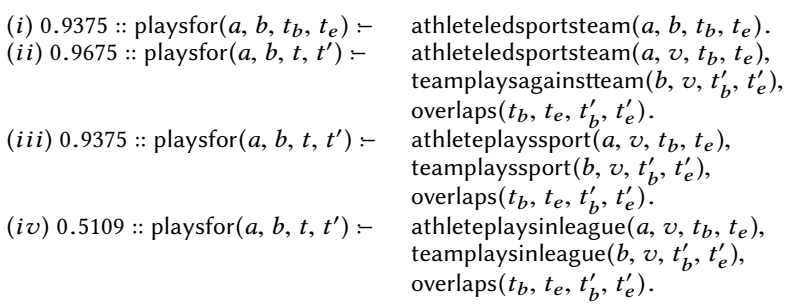

In order to perform reasoning tasks over probabilistic temporal facts and inference rules, in this study, we propose a bitemporal model for probabilistic knowledge graphs.

\section{KNOWLEDGE GRAPHS}

A knowledge graph is a set of triples that can be be encoded in the W3C standard RDF data model. Let I and L be two disjoint sets denoting the set of IRIs (identifying resources) and literals (character strings or some other type of data), respectively. We abbreviate the union of these sets $(I \cup L)$ as IL. A triple of the form $(s, r, o) \in \mathrm{I} \times \mathrm{I} \times \mathrm{IL}$ is called an RDF triple; $s$ is the subject, $r$ is the predicate or relation, and $o$ is the object of the triple. Each triple can be thought of as an edge between the subject and the object labeled by the predicate; hence a set of RDF triples is referred to as an $R D F$ graph. An RDF graph can be extended with temporal information by labeling each triple in the graph with a temporal element. For instance, the temporal element can represent the time period in which the triple is valid, i.e., the valid time of the triple $[12,13]$. In the next section, we extend knowledge graphs with the bitemporal model.

Schema and inference rules. Knowledge graphs often contain background knowledge to control and manage the quality of data and query answers. These background knowledge can be captured by first order logic. Web scale KGs often contain an ontology built using light weight languages such as RDFS (RDF Schema) and OWL2 (Web Ontology Language) profiles. OWL 2 profiles and RDFS are ontology languages used to express domain and range of relations, class and relations hierarchies among other constructs. Some of these languages can be represented by the Horn logic. In addition, ontology learning (also known as schema induction) is a new paradigm for automatically extracting inference/deduction rules from Web corpora. Most rule extraction systems produce Horn rules (that we call inference rules). For instance, Prob2FOIL [19], the SHERLOCK system [21] and ontological pathfinding (OP) [6] efficiently learn several thousands of first order Horn rules. These rules are expressive enough to represent complex relations in KG. We use the Datalog syntax to represent inference rules.

\section{BITEMPORAL KNOWLEDGE GRAPHS}

A bitemporal database is an extension of a conventional database by adding temporal element to each tuple in the database [16]. A tuple in a bitemporal database is timestamped with time intervals that represent the tuple's valid time and transaction time. Thus, a bitemporal database needs domains for two temporal universes, the valid time universe and the transaction time universe, and it may be desirable or convenient to restrict them to some subset of $\mathrm{T}$. Therefore, let $T_{v} \subseteq T$ denote the valid time universe of a bitemporal $\mathrm{KG}$, and $\mathrm{T}_{\mathrm{t}} \subseteq \mathrm{T}$ denote its transaction time universe. We consider a discrete time domain $T$ as a linearly ordered finite sequence of time points; for instance, days, minutes, or milliseconds. The finite domain assumption ensures that there are finitely many possible worlds in the probabilistic extension. A time interval is an ordered pair $\left[t_{b}, t_{e}\right)$ of time points, with $t_{b} \leq t_{e}$ and $t_{b}, t_{e} \in \mathrm{T}$, which denotes the closed-open interval of time points from $t_{b}$ to $t_{e}{ }^{1}$. We will work with the interval-based temporal domain for defining our data model. By combining bitemporal databases with knowledge graphs, we propose bitemporal knowledge graphs in Section 3.

${ }^{1}$ It is possible to extend to other interval based representations such as $\left[t_{b}, t_{e}\right]$, left and right closed interval. 
Multi-temporal RDF database models are first introduced by Grandi [12]. These models allow to capture several aspects of time in data such as validity, efficacy, transaction and so on. In this work, we consider validity and transaction times. A bitemporal KG has two temporal domains, the valid time domain and the transaction time domain. We restrict them to some subset of $\mathrm{T}$, i.e., let $\mathrm{T}_{\mathrm{v}} \subseteq \mathrm{T}$ denote the valid time universe of a $K G$, and $T_{t} \subseteq \mathrm{T}$ denote its transaction time universe. A valid time is the time period in which an RDF triple is considered true or valid. Transaction time is the time when a triple is added to a KG. Further, a bitemporal KG supports both transaction and valid time models.

Definition 3.1 (Bitemporal $K G$ ). A bitemporal $\mathrm{KG}$ is a $\mathrm{KG}$ where each fact $(s, r, o)$ in the graph is associated with a valid time $\left[v_{b}, v_{e}\right) \in$ $\mathrm{T}_{\mathrm{v}}$ and transaction time $\left[t_{b}, t_{e}\right) \in \mathrm{T}_{\mathrm{t}}$, i.e., $g=\left(s, r, o,\left[v_{b}, v_{e}\right),\left[t_{b}, t_{e}\right)\right)$. We refer to $g$ as a temporal fact and write it as a first order predicate $r\left(s, o, v_{b}, v_{e}, t_{b}, t_{e}\right)$. Right-unlimited time intervals are expressed as $\left[t_{b}, \mathrm{UC}\right)$ for transaction time and $\left[v_{b}\right.$, now) for valid time, where $\mathrm{UC}$ is short for Until Changed and now denotes the current time instance.

Moreover, we borrow two important notions, namely duplicatefree and coalescing, from temporal databases [8]. A bitemporal knowledge graph $G$ is called duplicate-free, if for all pairs of facts $r\left(s, o, v_{b}, v_{e}, t_{b}, t_{e}\right), r\left(s, o, v_{b}^{\prime}, v_{e}^{\prime}, t_{b}^{\prime}, t_{e}^{\prime}\right) \in G$, it holds that: [ $\left.v_{b}, v_{e}\right) \cap$ $\left[v_{b}^{\prime}, v_{e}^{\prime}\right)=\emptyset$ and $\left[t_{b}, t_{e}\right) \cap\left[t_{b}^{\prime}, t_{e}^{\prime}\right)=\emptyset$. In other words, if the nontemporal terms of two temporal facts are the same, then their temporal terms must be disjoint (non-overlapping). Temporal coalescing is the process of merging facts with identical non-temporal arguments and adjacent or overlapping time-intervals. This problem is especially challenging in the probabilistic setting. Performing coalescing is not straightforward because it is not clear what the probability of the new (coalesced) fact should be and it could be dependent on the application. We will investigate this issue in the next section.

EXAMPLE 1. We convert some of the facts of the knowledge graph in Table 1 into bitemporal facts as shown below. This is the same as aligning (or loosely merging) the NELL and Wikidata KGs for the task of knowledge base completion or bitemporal KG construction.

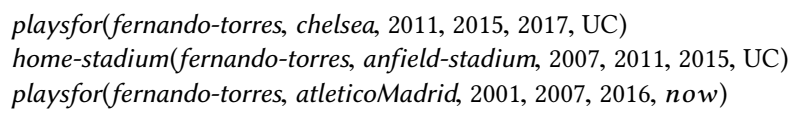

For the sake of presentation, we remove the date and month of the year and write just the years for both valid and transaction time intervals. Given a bitemporal KG $G$, its snapshot at time $t$ is the graph $G(t)$ (the non-temporal KG): $G(t)=\{(s, p, o) \mid(s, p, o,[t, t),[t, t)) \in$ $G$. The non-temporal KG associated with a bitemporal KG is $u(G)=$ $\bigcup_{t} G(t)$, the union of the graphs $G(t)$. Relying on this characterization, we define temporal entailment.

Definition 3.2 (Temporal entailment). We define temporal entailment as follows: for two bitemporal knowledge graphs $G_{1}$ and $G_{2}$, $G_{1} \quad=_{t} \quad G_{2}$ if and only if $G_{1}(t) \mid=G_{2}(t)$ for each $t$; $\mid=t$ denotes temporal entailment [13] and $=$ is the standard RDF entailment.

Temporalizing inference rules. The extraction of Horn rules with temporal constraints is notoriously difficult and has been afforded limited attention from the research community. However, the majority of the rules produced by rule learning systems can be converted into temporal rules by using the following: (i) add four variables $v_{b}, v_{e}, t_{b}$ and $t_{e}$ that represent time points of intervals to each predicate (i.e., $r(x, y)$ becomes $\left.r\left(x, y, v_{b}, v_{e}, t_{b}, t_{e}\right)\right)$ and (ii) if the number of predicates in the body is more than one, introduce an arithmetic predicate which is used to test temporal overlap, i.e.,

$$
r_{3}(x, z):-r_{1}(x, y) \wedge r_{2}(y, z)
$$

becomes

$$
\begin{gathered}
r_{3}\left(x, z, v, v^{\prime}, t, t^{\prime}\right):-r_{1}\left(x, y, v_{b}, v_{e}, t_{b}, t_{e}\right), r_{2}\left(y, z, v_{b}^{\prime}, v_{e}^{\prime}, t_{b}^{\prime}, t_{e}^{\prime}\right), \\
v=\min \left(v_{b}, v_{b}^{\prime}\right), v^{\prime}=\max \left(v_{e}, v_{e}^{\prime}\right), v_{b} \leq v_{e}^{\prime}, v_{b}^{\prime} \leq v_{e}, \\
t=\min \left(t_{b}, t_{b}^{\prime}\right), t^{\prime}=\max \left(t_{e}, t_{e}^{\prime}\right), t_{b} \leq t_{e}^{\prime}, t_{b}^{\prime} \leq t_{e}
\end{gathered}
$$

where $v, v^{\prime} \in\left\{v_{b}, v_{e}, v_{b}^{\prime}, v_{e}^{\prime}\right\}, t, t^{\prime} \in\left\{t_{b}, t_{e}, t_{b}^{\prime}, t_{e}^{\prime}\right\},\left[t, t^{\prime}\right)=\left[t_{b}, t_{e}\right) \cap$ $\left[t_{b}^{\prime}, t_{e}^{\prime}\right)$ and $\left[v, v^{\prime}\right)=\left[v_{b}, v_{e}\right) \cap\left[v_{b}^{\prime}, v_{e}^{\prime}\right)$. We refer to this process as temporalizing inference rules. Also, we refer to uncertain extensions of bitemporal KGs as probabilistic bitemporal KGs.

\section{PROBABILISTIC BITEMPORAL KNOWLEDGE GRAPHS}

As already discussed, a number of knowledge graphs contain probabilistic facts, some of them even include temporal information (either transaction time or valid time). In this section, we propose a data model for bitemporal probabilistic KGs.

\subsection{Data Model}

By annotating each triple in a knowledge graph with bitemporal as well as probabilistic information we obtain a probabilistic bitemporal KG. This allows us to model data where we are unsure about the time when it is true and record when the data was added to the KG. A probabilistic bitemporal KG contains a set of temporally annotated facts each with a confidence score (which can be a weight or probability value).

Definition 4.1 (Probabilistic bitemporal $K G$ ). A probabilistic bitemporal knowledge graph is a tuple $K=(G, F)$ with $G=\left\{\left(g, p_{1}\right), \ldots\right.$, $\left.\left(g_{n}, p_{n}\right)\right\}$ a temporal KG where each temporal fact $g_{i} \in G$ has probability $p_{i}$; and $F=\left\{\left(f_{1}, p_{1}\right), \ldots,\left(f_{m}, p_{m}\right)\right\}$ is a finite set of first order logic formulas representing background knowledge or schema and each $p_{i}$ denotes the probability of formula $f_{i}$.

In this paper, we restrict $F$ to be Horn formulas that express temporal inference rules and use the Datalog syntax to represent them. Given a possible world $K^{\prime}=\left(G^{\prime}, F\right) \subseteq K$, the probability of a $K^{\prime}$ is given by:

$$
P\left(K^{\prime}\right)=\prod_{G^{\prime} \cup F \mid{ }_{t} g} P(g) \cdot \prod_{\left.F \cup G \backslash G^{\prime}\right|_{t_{t}} g}(1-P(g)),
$$

where $\left.\right|_{t}$ denotes the temporal entailment relation. An important problem in temporal databases is coalescing, we investigate this problem in a probabilistic setting for bitemporal KGs and temporal queries.

\subsection{Coalescing}

Coalescing is a technique used in temporal databases for duplicate elimination $[2,8]$. Coalescing is useful for: reducing the size of probabilistic temporal KGs, and preventing incorrect answers in query evaluation. For instance, consider the query 'does fohn live in 


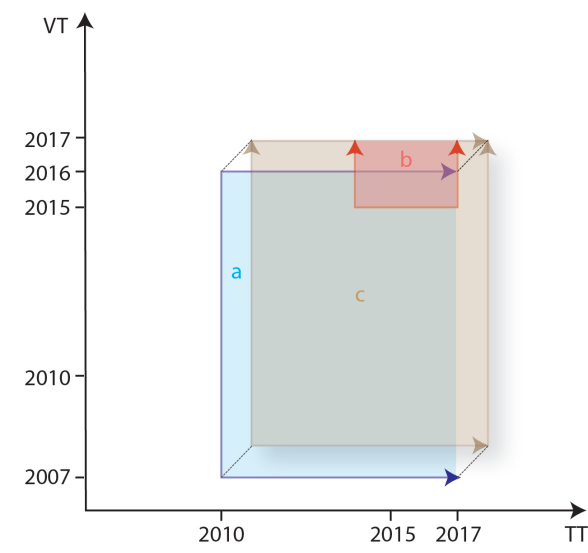

a 〈(John, livesIn, Paris, [2007, 2016), [2010, UC)), 0.9

b $\langle(J o h n$, livesIn, Paris, [2015, now), [2015, UC)), 0.5〉

c $\langle($ John, livesIn, Paris, [2007, now), [2010, UC)), 0.9

Figure 1: Coalescing probabilistic temporal facts. now indicates that the fact did not change whereas $U C$ denotes that the record has not be modified since its addition in the KG.

Paris from 2014 upto 2017?' on the temporal facts of Figure 1 before coalescing, i.e., (a) and (b). The result is no, however, the same query on the coalesced fact (c) (brown part of the figure), returns yes. Uncoalesced facts can arise in various cases: during query evaluation via projection or union operations, by not enforcing coalescing in update or insertion operations, and through information extraction from diverse sources or accuracy of the extractor. In this section, we discuss coalescing in probabilistic temporal KGs.

Definition 4.2 (Coalescing). Two probabilistic bitemporal facts $\left\langle r\left(s, o, v_{b}, v_{e}, t_{b}, t_{e}\right), p_{1}\right\rangle,\left\langle r^{\prime}\left(s^{\prime}, o^{\prime}, v_{b}^{\prime}, v_{e}^{\prime}, t_{b}^{\prime}, t_{e}^{\prime}\right), p_{2}\right\rangle$ can be coalesced if $s=s^{\prime}, r=r^{\prime}, o=o^{\prime}$ and the overlap of $\left[v_{b}, v_{e}\right)$ and $\left[v_{b}^{\prime}, v_{e}^{\prime}\right)$ (resp. $\left[t_{b}, t_{e}\right)$ and $\left.\left[t_{b}^{\prime}, t_{e}^{\prime}\right)\right)$ is non-empty. The probability $p_{3}$ of the coalesced fact $\left\langle r\left(s, o, v, v^{\prime}, t, t^{\prime}\right), p_{3}\right\rangle$ with $\left[v, v^{\prime}\right)=\left[v_{b}, v_{e}\right) \cup\left[v_{b}^{\prime}, v_{e}^{\prime}\right)$ $\left(\right.$ resp. $\left.\left[t, t^{\prime}\right)=\left[t_{b}, t_{e}\right) \cup\left[t_{b}^{\prime}, t_{e}^{\prime}\right)\right)$ is computed using one of the following three approaches.

Note that, in our proposal, in order to coalesce bitemporal facts, both the valid time and transaction time intervals must be overlapping. However, to calculate the probability of the coalesced fact, we only consider the overlap of the valid time intervals of the facts. We consider this assumption because the probabilities of temporal facts indicate that we are unsure about the time when they are true. The probability computations can be modified as needed or depending on the application, for instance, it is possible to assign the maximum of $p_{1}$ and $p_{2}$ as a probability to the coalesced fact.

4.2.1 Coalescing based on Allen's interval relations. In this approach, we use Allen's relations to coalesce facts and determine probabilities of the coalesced facts. The probability of the new coalesced fact can be the maximum or average of the facts that are to be merged, the choice depends on the application. Allen's relations are applied iteratively until closure.

4.2.2 Rule-based coalescing. One approach to coalescing is to use a iterative rule-based technique. In order to coalesce all the facts of a probabilistic temporal KG, we can construct Horn rules for each relation in the KG. Thus, rule-based coalescing can be done as follows: for each relation $r_{i}$ in a probabilistic temporal $\mathrm{KG} K$, apply the following rule iteratively until closure:

$$
\begin{gathered}
r_{i}\left(x, y, v, v^{\prime}, t, t^{\prime}\right):-r_{i}\left(x, y, v_{b}, v_{e}, t_{b}, t_{e}\right), r_{i}\left(x, y, v_{b}^{\prime}, v_{e}^{\prime}, t_{b}^{\prime}, t_{e}^{\prime}\right), \\
v=\min \left(v_{b}, v_{b}^{\prime}\right), v^{\prime}=\max \left(v_{e}, v_{e}^{\prime}\right), v_{b} \leq v_{e}^{\prime}, v_{b}^{\prime} \leq v_{e}, \\
t=\min \left(t_{b}, t_{b}^{\prime}\right), t^{\prime}=\max \left(t_{e}, t_{e}^{\prime}\right), t_{b} \leq t_{e}^{\prime}, t_{b}^{\prime} \leq t_{e} .
\end{gathered}
$$

The expression $t_{b} \leq t_{e}^{\prime}, t_{b}^{\prime} \leq t_{e}$ tests temporal overlap of the intervals $\left[t_{b}, t_{e}\right]$ and $\left[t_{b}^{\prime}, t_{e}^{\prime}\right]$. Besides, min, and max are predicates representing minimum, and maximum functions respectively. This approach uses one rule for each relation. If a KG has several hundreds of relations, we need the same number of coalescing rules to coalesce the KG. Hence, this operation can be very expensive, however, it is done only once.

EXAmple 2. Consider coalescing the probabilistic bitemporal facts, (a) and (b), shown in Figure 1 using the rule-based approach. This operation merges the two facts into one with the probability of the new fact (c) being the max of the two, i.e., $\max (0.9,0.5)$ which can also be learned upon inference.

4.2.3 Algorithmic coalescing. Another approach for coalescing probabilistic temporal facts is to use the following algorithm which is based on the results in [2]. In short, the algorithm continues as follows: for each relation $r$ search all temporal facts with the same non-temporal $(s, r, o)$ elements, order these facts according to their time period, for overlapping time periods build the maximum time period (union of time periods), delete the temporal facts whose time periods are contained in the maximum time period, and finally assign probability to the coalesced temporal facts. We leave out the comparison of the rule-based approach with that of the algorithmic as a future work.

\section{EXPERIMENTATION}

We conducted two different experiments: (i) marginal inference and (ii) MPE inference. For both experiments, we carried out performance tests in terms of running times. We run the experiments on a Debian 8 virtual machine with $2.6 \mathrm{GHz} 3$-core Intel Haswell processor, $24 \mathrm{~GB}$ of main memory and 1TB disk space.

Tools. We used the ProbLog system to carry out the experiments. ProbLog is a probabilistic extension of Prolog [15]. ProbLog uses a grounding (instantiating all the variables in the inference rules and queries with constants in all possible ways) technique that reduces the size of the number of ground rules significantly. Therefore, we chose ProbLog to perform the task of marginal and MPE inference. Data. For our experiments, we use the Wikidata knowledge graph. In particular, we extract a part of the KG that contains structured temporal information (obtained from various sources using open information extraction). We extracted temporal facts for various fluent relations including: playsFor, educatedAt, memberOf, occupation, spouse, and so on. Overall, we extracted over 6.7 million temporal facts from Wikidata. All of these temporal facts are interval timestampped (valid time). An excerpt of the data is shown below:

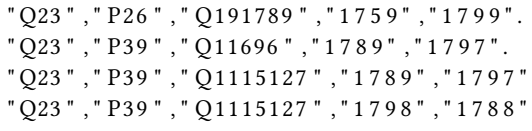


(a) Marginal and MPE inference.

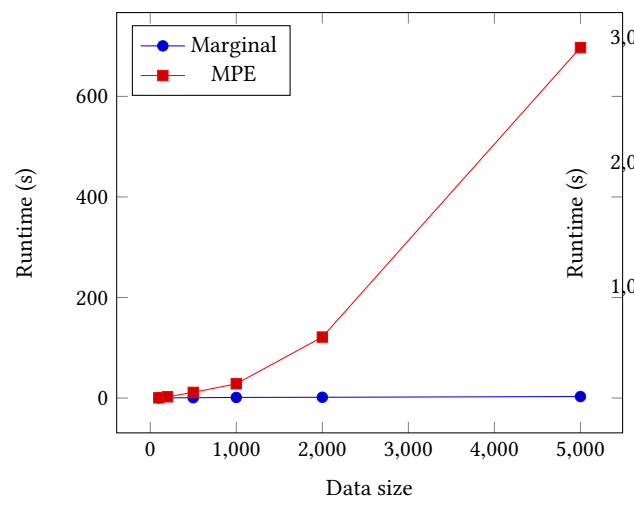

(b) Scalability test.

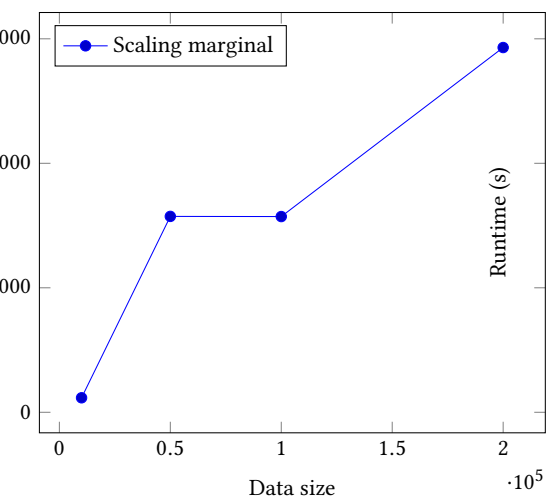

Runtime of grounding a KB in ProbLog

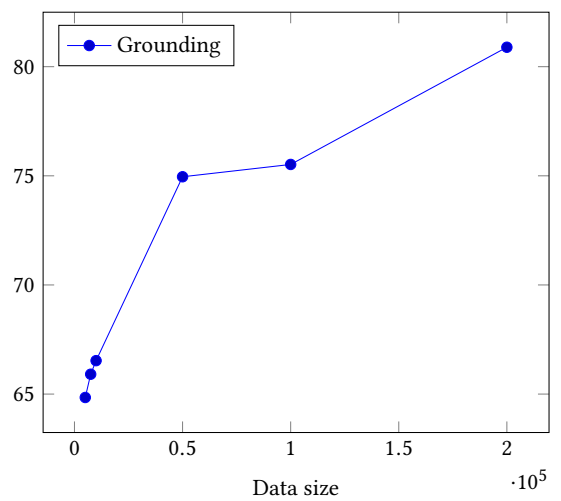

Figure 2: Run time performance of ProbLog over fixed query and varying data size.

"Q23 " , " P39 " , " Q1115127 " , " 1789 " , " 1797 ".

Q23, P26, and P39 are Wikidata shorthand for George Washington, spouse relation, and position held relation respectively. Besides, Q191789, Q11696, and Q1115127 are shorthand for Martha Washington, president of the USA, and commanding general of the USA respectively. The fourth and fifth columns represent the valid times of each relation. For example, the first line can be read as "George Washington's spouse was Martha Washington from 1759 upto 1799".

As Wikidata is a valid time knowledge graph, for our experiments, we extended it by adding transaction time intervals and probabilities to each fact in order to have a probabilistic bitemporal KG. We randomly generated transaction times and probabilities (> 0.5). Besides, as Wikidata is not complete, it does not contain valid times for all the facts. If the end time point of a fact is missing, we replace it with now. For instance, the end time (fifth column) of the following fact is missing: George Washington (Q23) obtained the military mark (P410) general of the armies of the USA (Q3100539) on 1976.

" Q23 " , " P410 " , " Q3100539 " , " 1976 " , " " ,

becomes

"Q23 ", , P410 " , " Q3100539 " , " 1976 " , " now " .

Temporal rules. We designed 36 different probabilistic temporal inference rules (ProbLog definite clauses) based on the fluents (time-varying relations) of Wikidata. The probabilities of the rules are generated randomly and are set between 0.5 and 0.99 . Some examples (in ProbLog syntax) of the rules is given below:

(i). The spouse (P26) relation is a sub property of the married relation.

$0.95:$ : married $(\mathrm{X}, \mathrm{Y}, \mathrm{Vb}, \mathrm{Ve}, \mathrm{Tb}, \mathrm{Te}):-\mathrm{P} 26(\mathrm{X}, \mathrm{Y}, \mathrm{Vb}, \mathrm{Ve}, \mathrm{Tb}, \mathrm{Te})$.

(ii). A person probably lives in a city where the university he educated at (P69) is located in (P131).

$$
\begin{array}{r}
0.5: \text { : livesin }(\mathrm{X}, \mathrm{Z}, \mathrm{V}, \mathrm{V}, \mathrm{Tb}, \mathrm{Te}):-\mathrm{P} 69(\mathrm{X}, \mathrm{Y}, \mathrm{Vb}, \mathrm{Ve}, \mathrm{Tb}, \mathrm{Te}), \\
\mathrm{P} 131(\mathrm{Y}, \mathrm{Z}, \mathrm{Vbp}, \mathrm{Vep}, \mathrm{Tbp}, \mathrm{Tep}), \mathrm{V} \text { is } \max (\mathrm{Vb}, \mathrm{Vbp}), \\
\mathrm{V} \text { is } \min (\mathrm{Ve}, \mathrm{Vep}), \mathrm{Vb} @<=\mathrm{Vep}, \mathrm{Vbp} @<=\mathrm{Tep} .
\end{array}
$$

Note that testing the overlap of the transaction time intervals is not done. This can be added if required. Another important point is what will be the transaction time of the newly inferred facts (livesin in our case). In the above rule, the transaction time of livesin is the intervals of the relation P69. However, this can also be set as needed, for instance, it can be [current date, UC).

\subsection{MPE Inference}

MPE inference is one of the most important tasks in probabilistic inference. It amounts to computing the most probable consistent bitemporal KG given evidence/data. In this experiment, we report the running times of ProbLog on different data sizes. The runtimes, averaged over 5 runs, are reported in Figure 2(a). As it can be seen, the runtime of ProbLog does not increase linearly with respect to the size of the input data. This is due to each added incorrect temporal fact might be involved in a conflict resulting in a non trivial optimization problem. In order to test the scalability of MPE inference, we increased the data size from $10^{4}$ upto $5 \times 10^{4}$. As expected, the running times were exceedingly high, i.e., upto several hours. When the datasize is 500000 , we interrupted the evaluation of MPE inference after a runtime of 3.63 hours. On the contrary, marginal inference scales quite well.

\subsection{Marginal Inference}

Marginal inference in probabilistic bitemporal KGs is the task computing the probability of a temporal query. In this experiment, we test the scalability of marginal temporal query evaluation. One of the bottlenecks of probabilistic query evaluation is finding efficient ways to generate a grounding of the rules and query. To tackle this problem, ProbLog applies relevant grounding by only grounding the rules that are relevant to a query. To test its efficiency, we carried out a performance experiment in terms of execution times. As shown in Figure 2(c), the run time of grounding the probabilistic bitemporal Wikidata KG, increases linearly with data size. However, this run time is dependent on the number of queries. If the number of queries is more than 100 , the run time of the grounding is very high. One approach to overcome this problem is to use a database engine to perform the grounding of inference rules and queries.

For marginal inference, the results of the experiment are reported in Figure 2(a). As shown, ProbLog performs very well. In comparison to MPE inference, the run time of marginal inference 
is almost constant when the size of the data is less than 10000 . This is because ProbLog performs approximate marginal inference as exact marginal inference is intractable. The reported runtimes are averaged over 5 runs. In order to test the scalability of marginal inference, we carried out additional experiments, reported in Figure 2(b). As shown, ProbLog computes the marginal probabilities of 20 queries over a data size of $10^{5}$ within 3000 seconds. These results are promising for probabilistic temporal query evaluation, however, ProbLog is not a suitable choice for MPE inference. Hence, we need to rely on other approximate inference engines such as DimmWitted.

\section{RELATED WORK}

Temporal databases have been extensively studied (see survey [22]). However, relatively few works exist on probabilistic temporal databases $[4,7,10]$. A relational database is used to model and query temporal data, integrity constraints and deduction rules can also be specified [10]. However, these rules must be deterministic (unweighted) unlike what we do here. On the other hand, contrary to this study where we use the bitemporal model, uncertain spatiotemporal databases focus on modelling trajectories through space and time.

Query evaluation in probabilistic databases is an active area of research. With respect to temporal query evaluation over a probabilistic temporal knowledge base, to the best of our knowledge, there are two important studies [5] and [10]. While the former focuses on MPE inference, we study here marginal inference and deal with the problem of temporal coalescing. The later deals with marginal inference, the difference with this work are the following: (i) we consider weighted inference rules and constraints, (ii) we propose coalescing for temporal $\mathrm{KBs}$, and (iii) we introduce rewriting for the coalescing of queries. In another study [11], the authors proposed an approach for resolving temporal conflicts in RDF knowledge bases. The idea is to use first-order logic Horn formulas with temporal predicates to express temporal and nontemporal constraints. However, these approaches are limited to a small set of temporal patterns and only allow for uncertainty in facts. Moreover, extending KBs using open domain information extraction, will often also lead to uncertainty about the correctness of schema information; a large variety of temporal inference rules and constraints, some of which will be domain specific, can also be the subject of uncertainty.

\section{CONCLUSION AND OUTLOOK}

In this work, we proposed a probabilistic bitemporal model for Web knowledge graphs. This model is relevant in order to record the extraction dates of probabilistic temporal facts as well as to support time travel queries. We have also addressed coalescing in a probilistic setting. Our preliminary experiments have shown that marginal inference (aka probabilistic query evaluation) scales very well. On the contrary computing the most probable bitemporal KG hardly scales. To overcome this problem, we will explore approximate query evaluation approaches. Hence, our future work has two directions: (i) to investigate the scalability of our approach, for instance by using highly scalable statistical approximate inference engines such as DimmWitted; and (ii) construct a bitemporal dataset by combining (aligning) the NELL and Wikidata datasets. This problem is known as knowledge fusion.

\section{REFERENCES}

[1] Sören Auer, Christian Bizer, Georgi Kobilarov, Jens Lehmann, Richard Cyganiak, and Zachary Ives. 2007. Dbpedia: A nucleus for a web of open data. The semantic web (2007), 722-735.

[2] Michael H. Böhlen, Richard T. Snodgrass, and Michael D. Soo. 1996. Coalescing in Temporal Databases. In VLDB'96, Proceedings of 22th International Conference on Very Large Data Bases, September 3-6, 1996, Mumbai (Bombay), India. 180-191.

[3] Andrew Carlson, Justin Betteridge, Bryan Kisiel, Burr Settles, Estevam R Hruschka Jr, and Tom M Mitchell. 2010. Toward an Architecture for Never-Ending Language Learning.. In $A A A I$, Vol. 5. 3.

[4] Melisachew Wudage Chekol. 2017. Scaling Probabilistic Temporal Query Evaluation. In Proceedings of the 2017 ACM on Conference on Information and Knowledge Management, CIKM 2017, Singapore, November 06 - 10, 2017. 697-706.

[5] Melisachew Wudage Chekol, Giuseppe Pirrò, Joerg Schoenfisch, and Heiner Stuckenschmidt. 2017. Marrying Uncertainty and Time in Knowledge Graphs. In Proceedings of the Thirty-First AAAI Conference on Artificial Intelligence, February 4-9, 2017, San Francisco, California, USA. 88-94.

[6] Yang Chen, Daisy Zhe Wang, and Sean Goldberg. 2016. ScaLeKB: scalable learning and inference over large knowledge bases. The VLDB fournal 25, 6 (2016), 893918.

[7] Alex Dekhtyar, Robert Ross, and VS Subrahmanian. 2001. Probabilistic temporal databases, I: algebra. ACM Transactions on Database Systems (TODS) 26, 1 (2001), 41-95.

[8] Anton Dignös, Michael H Böhlen, and Johann Gamper. 2012. Temporal alignment. In Proceedings of the 2012 ACM SIGMOD International Conference on Management of Data. ACM, 433-444.

[9] Xin Dong, Evgeniy Gabrilovich, Geremy Heitz, Wilko Horn, Ni Lao, Kevin Murphy, Thomas Strohmann, Shaohua Sun, and Wei Zhang. 2014. Knowledge Vault: A Web-Scale Approach to Probabilistic Knowledge Fusion. In SIGKDD. 601-610.

[10] Maximilian Dylla, Iris Miliaraki, and Martin Theobald. 2013. A temporalprobabilistic database model for information extraction. Proc. of the VLDB Endowment 6, 14 (2013), 1810-1821.

[11] Maximilian Dylla, Mauro Sozio, and Martin Theobald. 2011. Resolving Temporal Conflicts in Inconsistent RDF Knowledge Bases. In BTW. 474-493.

[12] Fabio Grandi. 2009. Multi-temporal RDF ontology versioning. In Proceedings of the 3rd International Workshop on Ontology Dynamics (IWOD-09).

[13] Claudio Gutierrez, Carlos Hurtado, and Alejandro Vaisman. 2005. Temporal RDF. In Proc. of European Semantic Web Conference. 93-107.

[14] Johannes Hoffart, Fabian M Suchanek, Klaus Berberich, Edwin Lewis-Kelham, Gerard De Melo, and Gerhard Weikum. 2011. YAGO2: exploring and querying world knowledge in time, space, context, and many languages. In Proceedings of the 20th international conference companion on World wide web. ACM, 229-232.

[15] Angelika Kimmig, Bart Demoen, Luc De Raedt, Vitor Santos Costa, and Ricardo Rocha. 2011. On the implementation of the probabilistic logic programming language ProbLog. Theory and Practice of Logic Programming 11, 2-3 (2011), 235-262.

[16] Anil Kumar, Vassilis J Tsotras, and Christos Faloutsos. 1998. Designing access methods for bitemporal databases. IEEE Transactions on Knowledge and Data Engineering 10, 1 (1998), 1-20.

[17] Xiao Ling and Daniel S Weld. 2010. Temporal Information Extraction.. In AAAI, Vol. 10. 1385-1390.

[18] Katerina Papaioannou and Michael Böhlen. 2016. TemProRA: Top-k temporalprobabilistic results analysis. In Data Engineering (ICDE), 2016 IEEE 32nd International Conference on. IEEE, 1382-1385.

[19] Luc De Raedt, Anton Dries, Ingo Thon, Guy Van den Broeck, and Mathias Verbeke. 2015. Inducing Probabilistic Relational Rules from Probabilistic Examples. In Proceedings of the Twenty-Fourth International foint Conference on Artificial Intelligence, IFCAI 2015, Buenos Aires, Argentina, Fuly 25-31, 2015. 1835-1843.

[20] Anisa Rula, Matteo Palmonari, Axel-Cyrille Ngonga Ngomo, Daniel Gerber, Jens Lehmann, and Lorenz Bühmann. 2014. Hybrid acquisition of temporal scopes for RDF data. In European Semantic Web Conference. Springer, 488-503.

[21] Stefan Schoenmackers, Oren Etzioni, Daniel S Weld, and Jesse Davis. 2010. Learning first-order horn clauses from web text. In EMNLP. 1088-1098.

[22] Richard T Snodgrass. 1992. Temporal databases. In Theories and methods of spatio-temporal reasoning in geographic space. Springer, 22-64.

[23] Partha Pratim Talukdar, Derry Wijaya, and Tom Mitchell. 2012. Coupled temporal scoping of relational facts. In Proceedings of the fifth ACM international conference on Web search and data mining. ACM, 73-82.

[24] Denny Vrandečić and Markus Krötzsch. 2014. Wikidata: a free collaborative knowledgebase. Commun. ACM 57, 10 (2014), 78-85. 